\title{
Touching Voids: On the Varieties of Absence Perception
}

\author{
Forthcoming in Review of Philosophy and Psychology \\ Dan Cavedon-Taylor \\ Brasenose College, University of Oxford
}

\begin{abstract}
Seeing one's laptop to be missing, hearing silence and smelling fresh air; these are all examples of perceptual experiences of absences. In this paper I discuss an example of absence perception in the tactual sense modality, that of tactually perceiving a tooth to be absent in one's mouth, following its extraction. Various features of the example challenge two recently-developed theories of absence perception: Farennikova's memory-perception mismatch theory and Martin and Dockic's meta-cognitive theory. I speculate that the mechanism underlying the experience is a body schema that has failed to update itself.
\end{abstract}

Keywords: absence perception; meta-cognition; touch; bodily experience

\section{I - Introduction}

Consider the following scenario:

Dentist You are at the dental surgery, undergoing a procedure to have a tooth removed. After the tooth's extraction, and once the anaesthetic wears off, you run your tongue along your teeth and arrive at the gap where once a tooth was located. The gap is experienced as unnerving, and not merely on its initial probing. For several days after the extraction you feel acutely unsettled whenever your tongue finds its way to the seemingly anomalous gap. That gap is not merely perceived as an empty space between two objects, i.e. the teeth that mark the boundaries of the gap. Rather, it is tactually perceived as a location at which a tooth is now 
missing. Something once experienced as present within your mouth is now experienced as lacking.

Dentist is an example of a perceptual experience, in the tactual modality, of an object's absence. It is a situation in which you have a tactual perception, via your tongue, of the absence in your mouth of an object that was once present: your tooth. The experience is properly perceptual, and not merely cognitive. You do not infer, after each probe, that the tooth is missing. You already knew that to be the case, having been awake during the procedure and having visually inspected the inside of your mouth, after the tooth's extraction, via the dentist's mirror. So the absence of the tooth is not something that you forgot and subsequently reconfirmed. Rather, on each haptic probe, in the days immediately following the procedure, you tactually re-experience the unnerving, slightly unsettling, absence of the now extracted tooth.

I shall argue here that this seemingly unremarkable experience constitutes a counterexample to two recent accounts of absence perception: Anna Farennikova's memory-perception mismatch model (sections II-IV) and Jean-Rémy Martin and Jérôme Dokic's meta-cognitive theory (section V). I speculate that the psychological mechanism responsible for the experience is a body schema that has failed to update itself (section VI).

It is worth contrasting the experience in Dentist with a closely related, albeit distinct experience. In the context of a discussion that concludes in favour of the existence of tactual sense-data, H.H. Price (1932) discusses cases in which absent objects are experienced as 'lingering' in the form of after-images.' Some of his examples are tactual: after one has removed one's hat, it may still feel present on one's head. Relatedly, in the case of phantom appendages, a body part that is literally absent continues to phenomenally linger after its amputation. The idea that one's extracted tooth is likewise an experiential 'lingerer' is a suggestive one and my positive account of the mechanism underlying the experience (see section VI) relies on the idea that the tooth is being represented as present by part of one's psychology.

Nonetheless, one should be careful not to think of the experience of objects as absent, and one should be careful not to think of Dentist in particular, as an experience of an object's after-image.

I am grateful to one of the journal's anonymous referees for drawing my attention to Price's discussion and for highlighting to me the possible connection between after-images and experiences of absence. 
For one, no argument for the existence of sense-data analogous to those one finds proceeding from considerations of after-images (see, e.g., O’Shaughnessy 2000: Ch.18) could proceed from cases of absence perception. After-images are appearances of objects, e.g., hats, lights, arms, etc. that linger in the absence of those objects. But perceptions of absence are not experiences of ghostly appearances or 'half-presences' akin to illusory experiences of pure visibilia like rainbows or shadows, as after-images are plausibly to be regarded (Phillips 2013). Feeling the absence of a tooth in one's mouth is not to be analysed as feeling a sort of shadowy, half-present tooth (mere tactibilia, if there could be such a thing). It is an experience of a thing's not being there at all.

Before beginning, I outline my assumptions about the nature of absence perception and perception more generally. First, I assume that tactual perception has high-level content (Siegel 2006) in that it represents not only low-level properties relating to spatial extent, texture, weight, etc., but also properties relating to the kind(s) under which tactually sensed objects falls under. For instance, running one's fingers along a table, one tactually represents the presence of a table (or perhaps wood), in addition to tactually representing the presence of variegated grooves on a relatively flat surface before one. One doesn't merely represent via touch that there is an object before one; touch takes a stand on the kind of object there. I assume that this carries over in cases of perceived absences, though matters here are delicate (see Farennikova 2013: 451-2). Thus, in Dentist and cases similar, one represents the absence of a tooth. Those who disagree can understand the content of one's tactual experience in Dentist along such lines as: that an object is absent. In making these assumptions, I also assume that perception does have content in that it represents the world as being some way. Tied up with the concept of representation is misrepresentation, since representations have accuracy conditions. Certainly, not everyone agrees that we should think of perception in this way (see Campbell 2002; Martin 2004 and Travis 2004), but insofar as I must adopt some standpoint from which to investigate absence perception, the representationalist one is my choice. Many assume that questions about perception easily translate between representationalist and non-representationalist frameworks. I make no such assumptions here (see Cavedon-Taylor 2015 for discussion). My final assumption concerns the scope of absence perception. Perceptual content has both an objectplace and a property-place. In hallucination, the accuracy conditions of the object-place are not met. In illusion, the accuracy conditions of the object-place are met, while those of the propertyplace are not. Accordingly, one can have perceptions of absences that are about the absence of certain properties and one can have perceptions of absences that are about the absence of certain objects. As should be clear from the above, my concern is with the latter. 


\section{II - The Memory-Perception Mismatch Theory}

Perceptual experiences of absence, like Dentist, occur in non-tactual modalities. Consider the following example:

Laptop You are working in your office, engrossed in writing a philosophy paper. You are called away to a meeting, in which you dutifully sit, itching to return to your work. The meeting ends and you make a beeline back to your office. You open the door, only to see your laptop is gone!

Taking cases like Laptop as central examples of absence perception, Farennikova (2013; 2015) makes a number of claims about the nature of such experiences. These claims are offered as non-modality-specific ones, and so are intended to provide a unified account of absence perception across the senses. The non-visual cases Farennikova mentions include:

- Hearing a lover's absence by hearing her footsteps grow quieter as she exits the building.

- Tasting the absence of chlorine in water.

- Smelling the absence of exhaust in the air.

- The sensation of missing a step while going down the stairs.

First, Farennikova (2013: 445) claims that the phenomenology of absence perception is one of incongruity. This rings true in both Laptop and Dentist. The perception of the laptop's absence and the perception of the tooth's absence both have a phenomenological 'oddness' to them. The experiences have a conscious character which might be described as one of strangeness, unfamiliarity and which are somewhat unsettling.

Second, Farennikova claims that the mechanism underlying absence perception is one of mismatch between images of objects ('object-templates') on the one hand and visual stimulus on the other. These object-templates are tokened in working-memory by the visual registering of contextual, environmental cues. These templates encode, sometimes only coarsely, the visible properties of objects. Once tokened, the template is 'projected' in order to be matched against further incoming perceptual stimuli. This projecting is described as "a process of holding up the template of a searched object for the purpose of comparison of its perceptual attributes with the 
attributes of the perceived scene." (Ibid: 441) If there is a mismatch, i.e. the projected template of $\mathrm{O}$ fails to refer to any perceived object in the scene, the result is that $\mathrm{O}$ is experienced as absent. As Farennikova puts it:

Visual experience of O's absence consists in an object-level mismatch between O's template generated by visual working memory and a percept of the observed stimulus.

If we couch this proposal in tactual terms, then we get the following:

Tactual experience of O's absence consists in an object-level mismatch between O's template generated by tactual working memory and a percept of the observed stimulus.

Such a model describes an "expectational mechanism." (Ibid: 440) You expected the presence of the laptop. What you saw was an empty desk. A perceptual experience of the laptop's absence ensued.

At this point, one might be tempted to think that the problem posed by Dentist is the following: when you first probed the inside of your mouth, after the extraction, you did not expect the tooth to be present. You believed the dentist to have removed the tooth. You looked in the mirror at the inside of your mouth, and actually saw the gap between the molars previously adjacent to the offending, now extracted, tooth. Moments later, when your tongue found its way to the gap, you did not think you would touch it again (that would have been unexpected). So it isn't that you expected to find the tooth to be present and were then surprised to find it absent. Moreover, it wasn't as if you had no expectations about the tooth's presence or absence. You did have expectations: you expected the tooth's absence and that is indeed what you found. So one might conclude that Farennikova's model simply does not apply in the case of Dentist, since there is no mismatch here between working-memory and occurrent tactual stimulus. There is, contrary to that model, a match. Still, we have the perception of an object's absence. Ergo, Farennikova's model falters in explaining Dentist. So we have a counterexample.

The above objection rests on a potential misunderstanding of Farennikova's model. The misunderstanding lies in thinking that the expectational mechanism described is a personal-level one, when it is, rather, advertised as a sub-personal one (Ibid: 440-4). For that reason, it seems that what one consciously expects or anticipates matters not, as far as the mechanism is 
concerned. Indeed, Farennikova is explicit that object-templates "may be projected involuntarily.” (Ibid: 442-3)

Still, the above line of objection cannot be said to miss the mark entirely. After all, there is a striking difference between Laptop and Dentist that needs explaining: how does the toothtemplate get tokened in working-memory, given that, unlike in Laptop, there is no personal-level expectation of the object's presence? Indeed, although the mismatch model is advertised as involving sub-personal expectations, some of the examples of absence perception Farennikova discusses seem to imply the presence of personal-level expectations:

You are about to make coffee and discover that the coffee jar is empty; or you expect an important document in the mail but there is nothing in your mailbox; or you make a trip to a bakery only to see that your favorite desert is missing from the display. These situations, in essence, are failed visual searches: you begin to look for an object, expecting it to be at a certain place, and see its absence when your expectation is disconfirmed. (Ibid: 440)

A very natural thought is that these are cases in which an agent consciously expects the presence of coffee, a particular document, a particular desert, etc. Thus, these examples plausibly depend upon the agent's having a personal-level expectation of an object's presence, one that is missing in the case of Dentist. Dentist would remain a counterexample that falls outside the scope of Farennikova's model.

Indeed, there is a further difficulty here for Farennikova, which is how the expectational mechanism can be sub-personal even in the case of Laptop. Farennikova claims that Laptop is a case of visual search:

When we expect something, we represent what is possible or likely in the environment. This advance information is coded as conditional on contextual cues. For instance, seeing a kitchen will cause you to expect to see a fridge and an oven. (Ibid: 440-1)

But if Laptop were a matter of conditional or contextual cues, then seeing any work-desk, in any office, should likewise occasion the perception of a laptop's absence. It wouldn't matter that one consciously expected the laptop's presence, one would have seen the laptop to be missing, irrespective of one's memory of it being located on the desk. If that is so, then there is an 
ambiguity in Farennikova's account over whether the expectations adverted to are personal or sub-personal. Moreover, this ambiguity forces the following dilemma: if the expectations are subpersonal, then while Dentist would pose little trouble, Laptop would seem to go unexplained. But if the expectations are personal-level, then they are missing in the case of Dentist, yet it is a case of a perceptual experience of absence.

\section{III - Deviant Patterns}

Farennikova's model has a great deal of flexibility. There are a number of ways it might be adapted to explain Dentist. Indeed, it is instructive to consider another example of absence perception in the visual modality, relatively similar to Dentist, and which Farennikova believes the mismatch model to explain: seeing the absence of a photograph when one reaches a gap in a series. On the face of it, this is similar to tactually experiencing the absence of the tooth, since the latter may be thought to involve running one's tongue along one's teeth before arriving at the experientially salient gap. On this account, both Dentist and seeing the absence of a photograph are experiences of what Farennikova calls 'deviant patterns': environmental regularities perceived at $t 1$ cue tokens of relevant object-templates in working-memory which then fail to match any stimuli perceived at $t 2$. For instance, as one visually experiences the photographs on the wall, a photograph-template is generated in working memory, as that is what is likely to come next. But when one arrives at the gap in the series, the template fails to match the incoming perceptual stimulus. What one ends up visually experiencing is not merely the presence of wall-space between two photographs; rather, one sees a photograph's absence. Similarly, Dentist might be explained by tactually experiencing a pattern of teeth, via one's tongue, and this likewise generating in working-memory an object-template of a tooth which then fails to match the stimulus encountered upon reaching the gap. If that is right, then Dentist is explained by the memory-perception mismatch model after all, and in such a way that treats the anticipatory mechanism at its core as automatic and sub-personal.

The difficulty is that there is no reason to think that the experience in Dentist depends upon moving one's tongue along the actually present teeth in this way. Granted, Dentist can be described in such a way that it is a case of perceiving a deviant pattern. But it would be dogmatic to insist upon this description since one may tactually perceive the tooth's absence in a number of ways that do not involve such patterns. First, one may simply press one's tongue directly onto 
the teeth adjacent to the gap. Alternatively, one may haptically probe the empty space itself. In the first situation, nothing resembling a pattern of stimulation enters the picture; there are simply two points of contact on the tongue, some small distance apart. In the second case, there is variation in stimulation as one actively explores the empty space, making contact with the two spatially separated teeth either side. But experiencing this variation in stimulation is nothing like seeing a line of photographs and suddenly coming to a deviant gap. It is more like staring at empty wall-space between two photographs and varying how one distributes one's attention between the two picture-frames.

\section{IV - Expected Absences}

I suggested above that one of the difficulties Dentist poses for Farennikova's model is that it is a case of experiencing an expected absence, and so seems to involve a fact of match between memorial expectation (i.e. the object-template) and perceptual stimulation. Crucially, however, Farennikova recognises that not all cases of absence perception are like Laptop in involving unexpected absences. Indeed, Farennikova claims the model allows for a disassociation between memory-perception mismatches and 'sensory surprises' (Ibid: 447), thereby allowing for experiences of absence that are expected. This is because what is central to the model is a contrast between the object-template in working memory and incoming perceptual stimuli:

[I]t is possible to see absences without any failure of expectation. Tourists traveling to a desert will expect to see no trees there. An observer will expect the sun to disappear behind the ocean line. Their expectations about absences... are accurate and upon confirmation will result in experiences of absence. (Ibid: 446)

It is not very obvious that these cases do constitute perceptual experiences of absences rather than inferrals of absences. But we can still test whether the model can be applied to Dentist. The idea would be that a perceptual experience of the tooth's absence occurs because, appearances to the contrary, there is a tooth-template in working-memory being matched against the tactually experienced gap. The explanation for why the template is tokened would be that, in line with Farennikova's theory, templates signal not only what objects one expects to be present but also what objects one expects to be absent (Ibid: 446). In Dentist, you expect the tooth to be absent, and this, no less than an expectation of its presence, tokens the relevant tooth-template in working 
memory. Indeed, on this view, the expected absence would be confirmed via the projecting of the template against incoming tactual stimulation of the gap between two teeth.

The potential problems with this explanation are threefold. First, it is not clear why one should think that expecting $\mathrm{O}$ to be absent in a scene involves the tokening of an object-template in memory, rather than the tokening of the belief that $\mathrm{O}$ is likely to be absent in that scene. Consider now the process of confirming that $\mathrm{O}$ really is absent. Need object-templates in memory be a necessary factor here? Farennikova claims so, though it is not obvious what supports this conjecture:

Suppose you learn that your colleague will not be attending the faculty meeting today, and so you come to expect her absence. You walk into the meeting room, and as you expected, she is not there. How do you confirm her absence? Presumably, you go through the same process of projection and mismatching. (Ibid: 447)

In Dentist you believe your tooth to be absent, having both testimonial and visual evidence of its removal. Why can't this belief simply be confirmed by inferences drawn from introspection of one's tongue-movements receiving no resistance at a particular spatial location?

Second, Farennikova's examples of expected absences, e.g., the experiences of the tourists and your experience of your absent colleague, do not seem to involve the phenomenology of incongruity. You expect your colleague to be absent from the meeting. Suppose you confirm that by sight and/or inferences from sight. Nothing feels amiss or anomalous in such a situation: what you see is what you expected. But this makes Farennikova's cases of expected absences entirely unlike those of unexpected absences like Laptop. This forces another dilemma for the memoryperception mismatch theory. The first horn: if the model can explain perceptual experiences of expected absences, as it is advertised to, then Farennikova is wrong about incongruity constituting the phenomenology of absence perception. Straightforwardly, the expected absences Farennikova discusses do not involve such a phenomenology. On the other horn: if Farennikova's model cannot explain perceptions of expected absences, then the model suffers counterexamples.

Third, and very much related to this last point, Dentist is a case of an expected absence that does involve the phenomenology of incongruity. This sets it apart from other examples of expected absences and makes it more like Laptop. Still, insofar as Dentist is a case of an expected absence 
it is unlike Laptop and more like the examples involving the tourists and your colleague. The problem is that one cannot explain all these cases in the same way: any account of the mechanism underlying experiences of expected absences, where those experiences lack the phenomenology of incongruity (e.g., seeing your colleague's absence), will ipso facto fail to account for expected absences that, like Dentist, do involve the phenomenology of incongruity.

But there is an alternative theory that concludes it is a mistake to think that the experience in Dentist is really perceptual at all: Martin and Dokic's (2013) 'meta-cognitive' account.

\section{V - The Meta-Cognitive Theory}

An alternative to the memory-perception mismatch model is the meta-cognitive theory. On this theory, developed by Martin and Dokic (2013), experiences of seeing one's laptop to be missing and feeling one's tooth to be absent are not really perceptual. Instead, they are properly described as 'meta-cognitive': any unusual phenomenology in such cases is nothing more than the feeling of surprise; in that respect, alleged cases of absence perception fail to involve a specifically 'perceptual' phenomenology of absence.

Martin and Dokic's argument for their conclusion involves reflection on a contrast case. In case 1, you are presented with 15 boxes. Upon opening them, you find a single red marble in each, at least for the first 10 boxes. Upon opening the $11^{\text {th}}$, you reveal a green marble. Case 2 is exactly the same, except box 11 has no marble. Martin and Dokic claim that a feeling of surprise is common to both cases, but that Farennikova must claim case 2 involves something further: a perceptual experience of the absence of a red marble. However, Martin and Dokic then argue that if we subtract from both cases one's surprise at not finding a red marble, there is nothing further to explain. There are only perceived presences of a green marble (in case 1) and the inside of a box (in case 2). If Martin and Dokic's theory is correct, we can explain all we need to explain about so-called 'absence perceptions' by simply talking about perceived presences and the meta-cognitive feeling of surprise.

But perceived presences, coupled with the feeling of surprise, cannot explain all that we need to explain about absence experiences. In particular, they cannot explain Dentist. For while surprise may be a factor in many cases of absence perception, it is not a factor that occurs in Dentist. 
Given that one expects the tooth's absence, what would be surprising would be for one to tactually experience the tooth's presence. Finding the tooth's absence is just what one expects. So, one is not surprised to then find the tooth absent. That is to say, what makes Dentist resistant to the meta-cognitive analysis is that, once again, it is an expected absence. This sets Dentist apart from seeing one's laptop, or a photograph, to be missing. Returning to the photography exhibit a second, or third, time, say, one now expects to see a gap between two photographs; it is plausible that one will no longer have an experience of a photograph's being absent and this will likely be matched by failing to feel surprised: one sees what one expects-the photograph isn't there. Ditto for experiencing the laptop's absence. But the experience in Dentist differs from both these. It occurs in the absence of any feeling of surprise. So, contrary to the meta-cognitive theory, the phenomenology of surprise is not central to any analysis of absence perception. Accordingly, the phenomenology of surprise ought to be kept distinct from the phenomenology of incongruity or anomalousness. Martin and Dokic blur this distinction, analysing the phenomenology of incongruity as surprise. But there is more nuance to incongruity than surprise. Dentist illustrates as much.

The meta-cognitive theorist may reply that they need not commit to a single analysis of all putative cases of absence perception: surprise is the correct analysis of some examples of absence perception, but different meta-cognitive feelings may need to be appealed to in order to explain others. The most obvious way to apply this strategy to Dentist would be to claim that all one needs to appeal to here is the perception of presences, i.e. the teeth still in one's mouth, plus a feeling of incongruity. This strategy mirrors what Martin and Dokic have to say about perceiving the empty box in case 2: one sees what is present, i.e. the inside of the box, and feels surprised.

The difficulty with this proposal is that it seems insufficient to capture the phenomenology of Dentist. For that phenomenology is not adequately captured by appealing to perceived presences, plus a feeling of incongruity. After all, it is not that what are perceived as present, the remaining teeth, are experienced as incongruous. Note the contrast with Martin and Dokic's case 2: plausibly, what is perceived as present there, the inside of the box, is genuinely surprising, given that all the other boxes were seen to contain marbles. But nothing that is perceived as present in Dentist is incongruous or anomalous. What is incongruous or anomalous is what is absent! Once one acknowledges this, it is difficult to refrain from taking the further step of affirming that the phenomenology in Dentist is perceptual, insofar as it is directed towards the gap. Given that one already believes there to be a gap there, such a phenomenology is not happily 
analysed as an instance of purely cognitive phenomenology either. The most natural explanation is that it is perceptual phenomenology. One has a tactual experience of a tooth's absence.

\section{VI - The Phantom Tooth}

All in all, we have reason to think that Dentist is a genuinely perceptual experience of an absence, contra Martin and Dokic, but one that fails to be explained by a memory-perception mismatch mechanism. What psychological mechanism does underlie Dentist? My speculative answer is that it involves a mismatch between body schema and incoming perceptual stimulation. In short, after the removal of the tooth, one's body schema fails to update itself. The result is that it continues to represent the tooth as present. When one moves one's tongue in one's mouth, one then has a tactual experience of the interior of one's mouth that conflicts with how it is represented by one's body schema; that is, as continuing to contain the extracted tooth. An unsettling tactual experience of the tooth's absence ensues. ${ }^{2}$

This account agrees with Farennikova's model insofar as it affirms that mismatching of representational content is the right way to account for Dentist. Given that the phenomenology of incongruity is present here, we have reason to think that a mismatch occurs somewhere in our psychology. But what makes Dentist unique among the examples of absence perception discussed here is that it is an example of experiencing the absence of one's own body part. (This, in part, is what makes the experience so acutely unnerving.) Taken together, these considerations strongly suggest that the mismatch at issue is between one's body schema, not working-memory, and tactual awareness of the inside of one's mouth. ${ }^{3}$

$2 \quad$ See Turker, et. al. (2005) for experimental evidence that the body schema encodes teeth. Moreover, those who argue for the possibility of incorporating extra-bodily tools into the body schema sometimes model such incorporation on the body's representation of teeth (Holmes and Spence 2006).

Of course, there might be other cases of perceiving the absence of one's own body part that are explained by Farennikova's model. One might see the absence of one's middle finger, following its amputation, say. Such an experience is plausibly explained by the perception of a deviant pattern, whereas Dentist need not be. 
One might wonder whether this proposal is compatible with Farennikova's model along the following lines: ${ }^{4}$ perhaps movement of the tongue in the direction of the missing tooth cues an object-template of the tooth in working-memory precisely in virtue of the body schema having failed to update itself. On this revised proposal, bodily movement triggers the tooth-template due to the body schema's continuing to represent the presence of the tooth.

By way of replying to this revised proposal, it will be helpful to reflect on differences between representation by working-memory versus representation by the body schema. This will also serve to further highlight important differences between Dentist and Laptop.

A key difference between body schema representation and working-memory representation is that the latter tends to update itself much faster than the former in response to incoming stimuli. (It is well-known that the body schema is relatively stable and can take significant time to update, resulting in experiences of so-called 'phantom' appendages.) Indeed, Farennikova claims that visual experience of the laptop's absence is fleeting and short-lived (Ibid: 445 n.12); it quickly dissipates into either a perceptual experience of the incoming stimulus (the presence of the desk) or collapses into visual imagery of the object-template (one's laptop). But as well as quickly dissipating, the experience in Laptop is unlikely to repeat itself; working-memory speedily updates how it represents the room in response to the incoming stimulus in such a way that one is not continually struck by the laptop's absence as one re-enters the room minutes, hours or days later. But this makes for a contrast with Dentist. First, the experience in Dentist is not fleeting and may be unsettling precisely because of its duration; one can experience the absence of the tooth for some time, as one haptically probes the void left by its extraction. Second, a few minutes, hours, mornings, etc., after the tooth's removal, one may continue to re-experience the tooth's absence in a way that one doesn't continue to re-experience the laptop's absence. Thus, considerations of duration and repeatability suggest that working-memory has little role to play in facilitating the tactual experience of absence in Dentist, whether in the way Farennikova's model suggests, or in the manner suggested by the revised view outlined above.

At the beginning of the paper, I attempted to distinguish experiences of after-images from experiences of absences. Phantom appendages, I mentioned, were thought of by Price (1932) to

\footnotetext{
I am grateful to one of the journal's anonymous referees for prompting me to think about this matter.
} 
be examples of the former. Yet hasn't what I have said here now blurred the line between the two? Experiences of phantom appendages are typically thought to be brought about in virtue of a body schema that has failed to match the actual organisation of one's body. But I have similarly relied upon this mechanism to explain the experience of absence in Dentist. How can these claims be maintained without tension?

The answer is that Dentist does not rely merely on a body schema that has failed to update itself. Rather, it relies on a mismatch between that body schema and tactual stimulation. ${ }^{5}$ Indeed, it is possible that subjects suffering with phantom appendages might undergo an experience similar to that in Dentist, if they haptically explore the space where they feel the phantom limb to be located. Still, assuming such a thing is possible, it would be the mismatch in tactual stimulation and body schema that would underlie this experience of the limb's absence. Crucially, it would not be a haptic sensing of a tactual after-image. So the two remain distinct.

\section{VII - Conclusion}

There is greater variety among our experiences of absence than is captured by the memoryperception mismatch and meta-cognitive theories. Attempting to unify phenomena by explaining them with a single model is a worthwhile endeavor, but by shoehorning too many cases into a single mold we risk overlooking genuine diversity. This, in turn, risks us missing out on a full and enriched understanding of the target explanandum, i.e. absence perception, and the myriad of mechanisms in the brain that might underlie it.

\footnotetext{
Another anonymous referee for this journal asks whether my view entails that a brain in a vat could have an experience of absence. My answer is 'yes.' One might find this result odd. For a brain in a vat, all objects are absent. So in having an absence experience, do I (and others in the debate) commit to brains in vats as having veridical experiences? Again, my answer is 'yes.' But this result is not so odd if one keeps matters of perceptual veridicality distinct from matters of perceptual contact with one's environment. On my view, the absence experiences of the brain in the vat are akin to veridical hallucination. The brain in the vat perceive objects to be absent which really are absent, hence such experiences are veridical. But the brain in the vat's absence experiences fail to involve the world itself in the relevant way that should cause us concern. For instance, qua brain in a vat, it fails to be in perceptual contact with the region of space between two teeth. This is the result we should want.
} 
Granted, there may be other examples of tactual experiences which are explained by both the memory-perception mismatch model and the meta-cognitive theory. ${ }^{6} \mathrm{My}$ claim is that there is at least one such example of a tactual experience, which, due to its being (i) expected, (ii) directed towards one's own body and (iii) having certain duration and repeatability, which is not well explained by these theories.

\section{Acknowledgements}

Parts of this paper were presented, in slightly different form, to an audience at the Institute of Philosophy in London. Thanks also to Roberto Casati, Jérôme Dokic, Roy Sorensen and Anna Farennikova for illuminating discussions of absence perception. Finally, this paper was improved by the written comments of Bence Nanay, Laura Gow and two of the journal's anonymous referees.

\section{References}

Campbell, J. (2002). Reference and Consciousness. OUP.

Cavedon-Taylor, D. (2015). "Kind Properties and the Metaphysics of Perception: Towards Impure Relationalism.” Pacific Philosophical Quarterly 96 (4): 487-509.

Farennikova, A. (2013). “Seeing Absence.” Philosophical Studies 166 (3): 429-454.

------. (2015). "Perception of Absence and Penetration from Experience.” Review of Philosophy and Psychology 6 (4): 621-640.

Holmes, N. \& Spence, C. (2006). "Beyond the Body Schema: Visual, Prosthetic, and Technological Contributions to Bodily Perception and Awareness.” In G. Knoblich, I. Thornton, M. Grosjean \& M Shiffrar (eds.), Human Body Perception From the Inside Out. Oxford University Press. 15-64.

Martin, J-R. and J. Dokic. (2013). “Seeing Absence or Absence of Seeing?” Thought 2 (2): 117125.

Martin, M. (2004). "The Limits of Self-Awareness.” Philosophical Studies 120 (1-3): 37-89.

For instance, suppose you reach into your bag to pull out your wallet (which you expected to be there), and you feel nothing but the inside of the bag. 
O'Shaughnessy, B. (2000). Consciousness and the World. OUP.

Phillips, I. (2013). “Afterimages and Sensation.” Philosophy and Phenomenological Research 87 (2): 417-453.

Price, H.H. (1932). Perception. Methuen.

Siegel, S. (2006). "Which Properties are Represented in Perception?" In T. Gendler \& J. Hawthorne (eds.), Perceptual Experience. Oxford University Press.

Travis, C. (2004). "The Silence of the Senses.” Mind 113 (449): 57-94.

Turker, K., Yeo P., \& Gandevia S. (2005). "Perceptual Distortion of Face by Local Anaesthesia of the Human Lips and Teeth.” Experimental Brain Research 165 (1): 37-43. 